\title{
The Medical Science Liaison Role in Spain: A Survey About the Opinion of HealthCare Professionals
}

\author{
Antonio González del Castillo ${ }^{1,2}$ (1) - Cristina García ${ }^{2,3} \cdot$ Adela Matesanz-Marín ${ }^{2,4} \cdot$ María José Gómez-Sánchez $^{2,5}$. \\ Victor Sastre ${ }^{2,6}$
}

Received: 25 May 2021 / Accepted: 13 August 2021 / Published online: 20 August 2021

(c) The Drug Information Association, Inc 2021

\begin{abstract}
Purpose/Aim There are limited studies addressing the knowledge of healthcare professionals (HCPs) on the Medical Science Liaison (MSL) role. The objective of this study was to determine the percentage of HCPs that know the role, and to describe their opinion about the utility of the MSL activities on their clinical practice.

Methods An online survey was completed by 107 HCPs between June 2019 and January 2020 through three Scientific Societies. It consisted of 17 or 25 questions, depending on the answers.

Results Most HCPs (73.8\%) knew the MSL role inside the pharmaceutical industry and were able to differentiate the MSL role from others in marketing/sales departments (77.6\%). Support for research projects $(70.8 \%)$ and training courses $(68.1 \%)$ were the most valuable activities. Participants scored the credibility of data provided by the MSL with a mean of 7.5 (out of 10 ), and the added value that the MSL provides to their clinical practice with a mean of 6.9. Among HCPs unfamiliar with the role (26.2\%), $60 \%$ of them are interested in meeting with the local MSL.

Conclusion Our study demonstrates that the MSL role is well-known by HCPs, mostly Key Opinion Leaders, and they agree on the importance of working together in scientific projects on, e.g., disease awareness, medical training, or clinical trials. Given the advance of targeted therapies and the move toward personalized medicine, the MSL role will be in more demand and necessary for HCPs in the years to come.
\end{abstract}

Keywords Medical science liaison $\cdot$ Healthcare professionals $\cdot$ Survey $\cdot$ Opinion $\cdot$ Spain

\section{Introduction}

The Medical Science Liaison (MSL) is a specialized role within pharmaceutical, biotechnology, medical devices, diagnostic companies, and other healthcare industries [1].

Antonio González del Castillo

antonio.gonzalezdc@gmail.com

1 MSL Medical Department, Sanofi Genzyme, Calle de Martínez Villergas, 52, 28027 Madrid, Spain

2 MSL Profile Working Group, Medicine Association of the Pharmaceutical Industry (AMIFE), Madrid, Spain

3 Medical Affairs Department, Persan Farma, Las Palmas de Gran Canaria, Spain

4 MSL Medical Department, Chiesi España S.A., Barcelona, Spain

5 Medical Department Exeltis, Madrid, Spain

6 Medical Department, Amgen, Barcelona, Spain
MSL professionals frequently have advanced training such as $\mathrm{PhD}$, PharmD, or $\mathrm{MD}$, and cover a wide range of therapeutic areas, such as Oncology, Neurology/Neuroscience, Rare Diseases, Immunology, Cardiology, Hematology, Endocrinology, and Dermatology [1]. The number of MSLs inside the industry is increasing quickly. According to the MSL Society, MSLs will grow in approximately $20 \%$ over the following 1-2 years [2]. Since its establishment in 1967 by the Upjohn Corporation, the MSL role has changed, rising in scope and activities [3, 4]. The essential objective is to establish and maintain peer-to-peer relationships with healthcare professionals (HCPs) and key opinion leaders (KOLs) [1]. KOL are characterized by being well-known in their research field and academic environment [5]. Additionally, KOLs act as officers or speakers at both national and international meetings. The MSL role is non-promotional; different and separated from sales and marketing functions [6]. The MSL aims to be an impartial expert with whom key HCPs can obtain updated safety and efficacy data 
about therapeutic agents and diseases, especially with the increasing use of biological agents and an increased interest in personalized medicine. The scientific exchange is twoway, and the collected information can impact forthcoming activities of the companies and address unmet local needs [6]. The MSL-HCP relationship aims to provide added value to multidisciplinary clinical management [2]. To date, limited studies have been published on the MSL role (experience, responsibilities, value demonstration) addressing the knowledge of the HCP about the MSL [2, 3, 7-17]. The MSL profile project in Spain emerged out of the MSL task force of the Association of Medicine of the Pharmaceutical Industry in Spain (AMIFE, 'Asociación de Medicina de la Industria Farmacéutica en España') at the end of 2017, and took shape during 2018. It aimed at establishing the MSL profile through the opinion of different stakeholders from the pharmaceutical and healthcare sector: MSL, medical and research departments (medical affairs, medical information, clinical operations, and pharmacovigilance), sales/marketing departments, and HCPs. The present study represents the fourth of the surveys involved (one of them already published [18] and two pending publication), where specific objective was to determine the percentage of HCPs that know the MSL role and describe their opinion about the utility of the MSL activities on their clinical practice.

\section{Materials and Methods}

\section{Study Design}

The survey was specifically developed by the MSL task force of AMIFE based on the literature [14]. The survey consisted of 17 or 25 questions depending on the response to question 15 (Table 1). The time for completing it was approximately $10 \mathrm{~min}$. Three Spanish Scientific Societies, i.e., the Spanish Diabetes Society (SED, 'Sociedad Española de Diabetes'), the Spanish Society for Bone and Mineral

Table 1 Survey questions

\begin{tabular}{|c|c|}
\hline 1 & Which autonomous community (region) do you work in? \\
\hline 2 & Which position do you have? \\
\hline 3 & What specialty do you have? \\
\hline 4 & Have you published in international journals over the last 10 years? \\
\hline 5 & Do you usually attend congresses? \\
\hline 6 & Have you participated in clinical trials? \\
\hline 7 & Do you participate actively in a scientific society (board member, task force, meetings organization, or others)? \\
\hline 8 & Do you know the difference between the promotional and non-promotional information of medications? \\
\hline 9 & How many pharmaceutical companies have visited you over the last year? \\
\hline 10 & Do you differentiate the MSL role from other figures in marketing/sales departments? \\
\hline 11 & Are you in touch with sales representatives? \\
\hline 12 & Are you in touch with marketing departments? \\
\hline 13 & Are you in touch with a MSL of your area? \\
\hline 14 & $\begin{array}{l}\text { Are you in touch with other figures of the medical department (different to MSL, i.e., medical advisor, medical } \\
\text { manager, medical director, etc.)? }\end{array}$ \\
\hline \multirow[t]{2}{*}{15} & Do you know the MSL role inside the pharmaceutical industry? \\
\hline & If NO: \\
\hline 16 & Would you be interested in meeting the MSL of your area? \\
\hline \multirow[t]{2}{*}{17} & What type of information would you request? \\
\hline & If YES: \\
\hline 16 & Assess the utility of promotional activities in your clinical practice \\
\hline 17 & Assess the utility of non-promotional activities in your clinical practice \\
\hline 18 & Assess the utility of activities not associated with the industry in your clinical practice \\
\hline 19 & How long does each session with the MSL usually last? \\
\hline 20 & Assess the credibility of data provided by the MSL \\
\hline 21 & Assess the added value that the MSL provides to your clinical practice \\
\hline 22 & Do you usually request medical information? \\
\hline 23 & Who usually send it (the medical information) to you? \\
\hline 24 & What type of information would you request? \\
\hline 25 & Have you attended an online session with the medical department? \\
\hline
\end{tabular}

$M S L$ medical science liaison 
Metabolism Research (SEIOMM, 'Sociedad Española de Investigación Ósea y del Metabolismo Mineral'), and the Spanish Society of Medical Oncology (SEOM, 'Sociedad Española de Oncología Médica') were responsible for the recruitment of participants, who were all physicians. The boards of directors of the Scientific Societies evaluated the survey and approved the use of the survey for their members. These societies invited all their members to participate in the survey (SEIOMM and SED by direct mail in October 2019 and January 2020, respectively; and SEOM via the June 2019 Newsletter). Being an HCP was the only criterium to participate in the study. There were no exclusion criteria. The answers to the questions were either yes/no or multiplechoice. The survey was available online (SurveyMonkey. com), and it was completed anonymously.

\section{Quantitative and Qualitative Assessments}

The opinion of HCPs about the utility of MSL activities on their clinical practice was evaluated with one of the following responses: none, low, moderate, or high. Credibility and added value were quantified using a $0-10$ color range indicator (red: 0-4 score, yellow: 5-7, and green: 8-10). Higher values represented greater credibility and added value.

\section{Statistical Analysis}

Quantitative responses were expressed as the mean and the standard deviation (SD), and qualitative ones as absolute and relative frequencies. Results were evaluated overall and in subgroups, depending on if the responder was or was not familiar with the MSL role as well as their years of clinical experience. Comparisons of variables between groups were carried out with chi-square or t-test, when appropriate. The statistical significance was established with $p<0.05$. All statistical analyses were conducted with SAS 9.4 software.

\section{Results}

\section{Characteristics of Participants}

A total of 107 HCPs completed the survey, representing the majority of regions from Spain, especially Catalonia (25.7\% of respondents), Community of Madrid (16.2\%), and Andalusia (13.3\%). Of them, $54.2 \%$ were members of the SED, $36.4 \%$ of the SEIOMM, and $9.3 \%$ of the SEOM. Participants were associate doctors (having more than 10 years of experience; $43.4 \%$ of total, being heads of department up to $12.3 \%)$, endocrinologists or rheumatologists $(42.7 \%$ and $21.4 \%$, respectively), who had publications in international journals in the last 10 years $(58.9 \%)$, frequently attended national or international congresses $(86.0 \%$ and $69.2 \%)$, participated in clinical trials $(82.2 \%)$, as a principal investigator $(41.1 \%)$ or collaborator $(66.4 \%)$, and were actively involved in a national scientific society $(46.7 \%)$. Most of respondents $(67.3 \%)$ knew the difference between promotional and non-promotional information on medications and received a visit of more than ten pharmaceutical companies over the previous year $(71.0 \%)$. The majority of participants (77.6\%) differentiated the MSL role from other roles in marketing/sales departments, and also knew the MSL role inside the pharmaceutical industry $(73.8 \%)$. Characteristics of HCPs are classified depending on their familiarity with the MSL role. In Table 2, awareness of MSLs is characterized by level of HCP experience. Table 3 illustrates how often these HCPs interacted with pharmaceutical companies and their interaction with marketing and/or sales representatives or other pharmaceutical representatives.

\section{HCPs Who were Familiar with the MSL Role}

There were 79 HCPs who knew the MSL role. The profile of these HCPs in comparison with those who did not know the MSL role was characterized by having more publications during the last 10 years $(64.6 \%$ vs $42.9 \%, p=0.045)$ and greater participation in clinical trials $(89.9 \%$ vs $60.7 \%$, $p=0.0005$, Table 2). Moreover, these HCPs knew the difference between promotional and non-promotional information ( $75.9 \%$ vs $42.9 \%, p=0.004)$. In addition, they were visited by more than ten pharmaceutical company representatives during the last year $(78.5 \%$ vs $50 \%, p=0.016)$ and had more contact with marketing and medical departments $(79.7 \%$, $p=0.0026$ and $71.4 \%, p=0.007$, respectively), including MSLs (91.0\%, $p<0.0001$, Table 3$)$. They clearly differentiated the MSL role $(93.7 \%, p<0.0001)$.

Activities with highest utility for HCP's clinical practice were: attendance at congresses (72.6\%); support for research projects by the medical department $(70.8 \%)$; training courses or stays in reference centers (68.1\%); and provision of medical literature and studies published on PubMed (68.5\%, Fig. 1). When comparing the utility valuation of the different activities conducted by the MSL, HCPs considered clinical sessions, roundtables organized and virtual meetings, more valuable than promotional meetings $(p=0.0001, p<0.0001$ and $p=0.0091$, respectively).

Participants scored the credibility of data provided by the MSL with a mean of 7.5 (SD: 1.7), and the added value that the MSL provides to the clinical practice with a mean of 6.9 (SD: 2.2; Fig. 2). Face-to-face interactions with the MSL predominantly (65.7\% of respondents) lasted less than $10 \mathrm{~min}$. Despite the survey being conducted before the COVID-19 pandemic, a total of $53.4 \%$ of participants stated that they have attended an online session with the medical department. When asked about the type of information that was of most interest, $74.6 \%$ and $66.7 \%$ of respondents requested updated 
Table 2 Characteristics of healthcare professionals depending on their clinical experience and their familiarity with the MSL role

\begin{tabular}{|c|c|c|c|}
\hline & \multicolumn{2}{|c|}{ Familiar with the MSL role } & \multirow[b]{2}{*}{$p$ value } \\
\hline & Yes $(N=79)$ & No $(N=28)$ & \\
\hline Position, $n(\%) *$ & & & 0.219 \\
\hline Medical resident & $2(2.5)$ & $2(7.4)$ & \\
\hline Associate doctor $(<5$ years $)$ & $8(10.1)$ & $6(22.2)$ & \\
\hline Associate doctor (5-10 years) & $19(24.1)$ & $2(7.4)$ & \\
\hline Associate doctor (>10 years) & $35(44.3)$ & $11(40.7)$ & \\
\hline Head of department & $10(12.7)$ & $3(11.1)$ & \\
\hline Private medicine & $5(6.3)$ & $3(11.1)$ & \\
\hline $\begin{array}{l}\text { Publications in the last } 10 \text { years in international } \\
\text { journals, } n(\%)\end{array}$ & & & 0.045 \\
\hline Yes & $51(64.6)$ & $12(42.9)$ & \\
\hline No & $28(35.4)$ & $16(57.1)$ & \\
\hline \multicolumn{4}{|l|}{ Frequent attendance to congresses, $n(\%)$} \\
\hline No & $0(0.0)$ & $1(3.6)$ & \\
\hline Yes, regional ones & $48(60.8)$ & $18(64.3)$ & \\
\hline Yes, national ones & $66(83.5)$ & $26(92.9)$ & \\
\hline Yes, international ones & $58(73.4)$ & $16(57.1)$ & \\
\hline Habitual participation in clinical trials, $n(\%)$ & & & $<0.001$ \\
\hline No & $8(10.1)$ & $11(39.3)$ & \\
\hline Yes, as a collaborator & $57(72.2)$ & $14(50.0)$ & \\
\hline Yes, as a principal investigator & $34(43.0)$ & $10(35.7)$ & \\
\hline Active involvement in a scientific society, $n(\%)$ & & & 0.345 \\
\hline No & $26(32.9)$ & $12(42.9)$ & \\
\hline Yes, at regional level & $28(35.4)$ & $8(28.6)$ & \\
\hline Yes, at national level & $39(49.4)$ & $11(39.3)$ & \\
\hline Yes, at international level & $6(7.6)$ & $1(3.6)$ & \\
\hline
\end{tabular}

$M S L$ medical science liaison

*Information about the position was missing in one healthcare professional who was unfamiliar with the MSL role safety and efficacy information of commercialized products, respectively (Fig. 3).

\section{HCPs Who were Unfamiliar with the MSL Role}

Of participants, 28 (26.2\%) stated not knowing the MSL role inside the pharmaceutical industry. Among them, $16 \mathrm{HCPs}$ were associate doctors ( $>5$ years) or head of departments (Table 2). Overall, $60 \%$ of these participants were interested in knowing the MSL in their therapeutic area, response that was positively increased among those HCPs with more experience up to $71.4 \%$. A total of $56.0 \%$ and $68 \%$ requested updated efficacy and safety information of commercialized products, respectively (Fig. 3).

\section{Discussion}

As part of four surveys depicting the opinion of important stakeholders in the pharmaceutical and healthcare sector [18], our present study presents the knowledge of the HCP about the MSL role. Overall, most HCPs knew of the MSL (73.8\%). Moreover, they pointed out to high credibility (7.5 out of 10) and added value (6.9 out of 10) from the MSL role and highlighted some MSL activities, such as training courses or support to research projects. In addition, $60 \%$ of HCPs who did not know the MSL role were interested in meeting the MSL in their area. 
Table 3 Relationship and knowledge of healthcare professionals with pharmaceutical companies depending on their familiarity with the MSL figure

\begin{tabular}{|c|c|c|c|}
\hline & Familiar wit & e MSL role & \\
\hline & Yes $(N=79)$ & No $(N=28)$ & $p$ value \\
\hline Number of pharmaceutical companies that have visited you over the last year, $n(\%)$ & & & 0.016 \\
\hline $1-5$ & $7(8.9)$ & $5(17.9)$ & \\
\hline $6-9$ & $10(12.7)$ & $9(32.1)$ & \\
\hline$>10$ & $62(78.5)$ & $14(50.0)$ & \\
\hline Do you differentiate the MSL role from other figures in marketing/sales departments?, $n(\%)$ & & & $<0.001$ \\
\hline Yes & $74(93.7)$ & $9(32.1)$ & \\
\hline No & $5(6.3)$ & $19(67.9)$ & \\
\hline Are you in touch with sales representatives?, $n(\%)$ & & 0.106 & \\
\hline Yes & $78(98.7)$ & $26(92.9)$ & \\
\hline No & $1(1.3)$ & $2(7.1)$ & \\
\hline Are you in touch with marketing departments?, $n(\%)$ & & & 0.003 \\
\hline Yes & $63(79.7)$ & $14(50.0)$ & \\
\hline No & $16(20.3)$ & $14(50.0)$ & \\
\hline Are you in touch with a MSL of your area?, n (\%)* & & & \\
\hline Yes & $71(91.0)$ & $8(28.6)$ & \\
\hline No & $7(9.0)$ & $20(71.4)$ & \\
\hline $\begin{array}{l}\text { Are you in touch with other figures of the medical department (different to MSL, i.e., medical } \\
\text { advisor, medical manager, medical director, etc.)?, } n(\%) * *\end{array}$ & & & 0.007 \\
\hline Yes & $55(71.4)$ & $12(42.9)$ & \\
\hline No & $22(28.6)$ & $16(57.1)$ & \\
\hline Do you know the MSL role within the pharmaceutical industry?, $n(\%)$ & & & NA \\
\hline Yes & $79(100.0)$ & $0(0.0)$ & \\
\hline No & $0(0.0)$ & $28(100.0)$ & \\
\hline
\end{tabular}

MSL medical science liaison, $N A$ not available

*Information about being in touch with the MSL was missing in one healthcare professional with low clinical experience and who was familiar with the MSL figure

**Information about being in touch with other figures of the medical department was missing in two healthcare professionals with high clinical experience and who were familiar with the MSL figure

Despite the significant expansion of the MSL role during the last years inside the pharmaceutical and healthcare companies, limited literature addressing the knowledge and valuation of the HCPs on the MSL role has been published [2, 3, 7-17]. Moss et al. [13] reported the expectations of 116 KOLs on MSLs in the diabetes therapeutic area. Of them, $34 \%$ of academic $(n=29)$ and 55\% practicing KOLs ( $n=87$ ) pointed to the MSL when asked about the source of acquiring knowledge useful in their clinical practice. Product specific education, disease state education, and research opportunities were the most frequent services received from the MSL. Among abilities and attributes of the MSL, academic and practicing KOLs highlighted: to provide unbiased product comparisons, to act in an ethical manner, and the ability for addressing safety issues. In contrast with Moss et al. [13] our study was focused on differentiating HCPs who know the MSL role from those who do not so, and then evaluate the role based on their activities. Similarly, to this publication, the activities most valuable were those related with training courses and support to research projects. The MSL role is one of the most important sources of information for HCPs/KOLs. This observation is aligned with Moss et al. [13] which approximately a third of academic KOLs and half of practicing KOLs rated the MSL as a first or second source of important information. In another survey with the opinion of 296 KOLs from diverse therapeutic areas, Moss et al. [3] showed top attributes expected of MSL (product knowledge, scientific credibility, therapeutic area knowledge, timeliness of responsiveness, and communication skills) to support curriculum development in advanced MSL training. In line with this, our study has also highlighted the need for information that HCPs/KOLs request of the MSL, mainly updated safety, efficacy, and non-label use of drugs. Attributes and skills, together with the tendency toward personalized medicine and the complexity of treatments, have led to an increased demand of the number of 


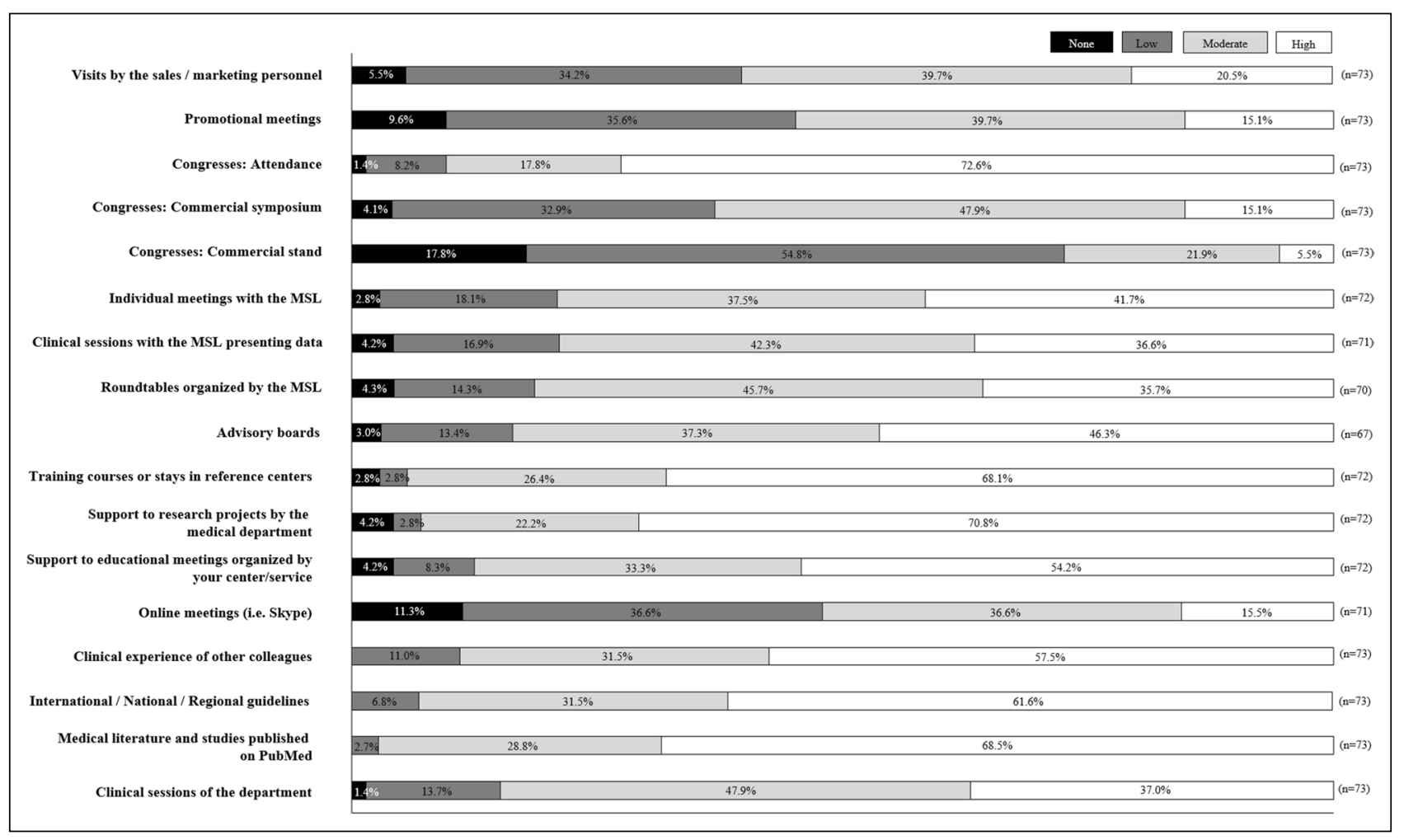

Fig. 1 Utility of diverse activities for HCP's clinical practice

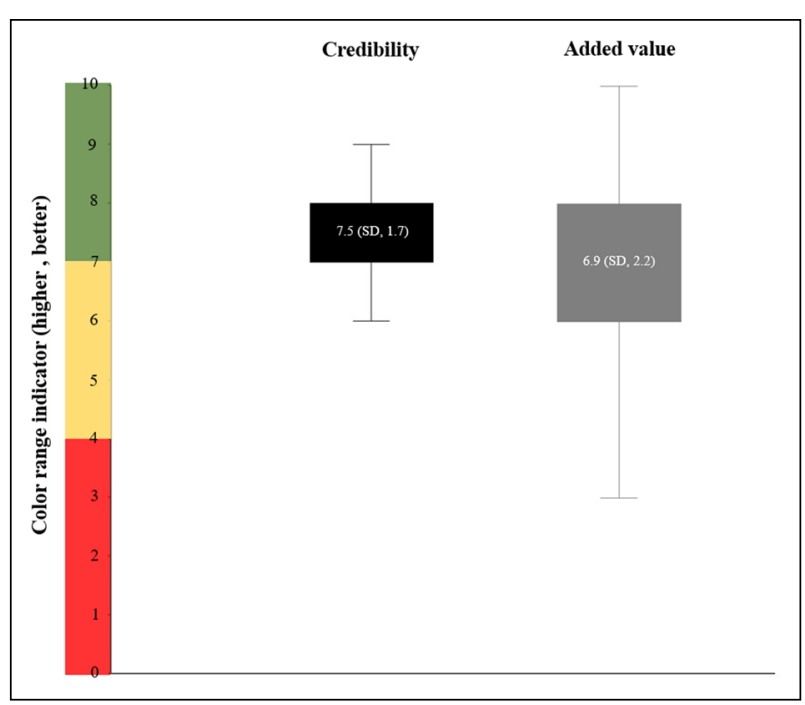

Fig. 2 Credibility and added value of the MSL. Values inside the boxes are the mean score and the standard deviation (SD)

MSL in healthcare companies [2]. The need for personalized information could be driver for the increase of clinical sessions or meetings with an MSL instead of promotional meetings.

It is remarkable that one of the most valuable activities from the MSL, support to research projects (70.8\%), was not one of the priorities when HCPs were asked what information they would request (36.5\%). There is not a confirmed response about why it happened, but we are wondering if HCPs/KOL are really aware about this capability from the MSLs. Although we know that it is not easy to support the research, there are multiple examples of how MSLs have contributed to enhance different investigator initiated trials (ITT) [16]. Furthermore, it was interesting to find that HCPs/ KOL stated that the time they dedicated to the MSLs was less than $10 \mathrm{~min}$. Not only from our perceptions but also due to the MSL's engagement in face-to-face meetings, so they tend to last longer. Maybe the question is not well written, or the responses are very bounded. Further information in this regard should be collected in future studies. Lastly, we found that although more than half HCPs/KOLs had attended virtual meetings, only $35.6 \%$ were satisfied and $15.5 \%$ considered it an important activity for the MSLs. These results are in line with a previous survey by Sastre et al. [18]. Authors showed that although up to $65 \%$ of the participants used virtual interactions, $13 \%$ were dissatisfied because of technical issues. It should be noted that the present survey was conducted prior to the COVID-19 pandemic. Therefore, the responses might have varied if evaluated during or after it. Indeed, the MSL Society carried out in September 2020 an online survey on how KOLs prefer to engage with MSLs during the pandemic and showed that engagements with 


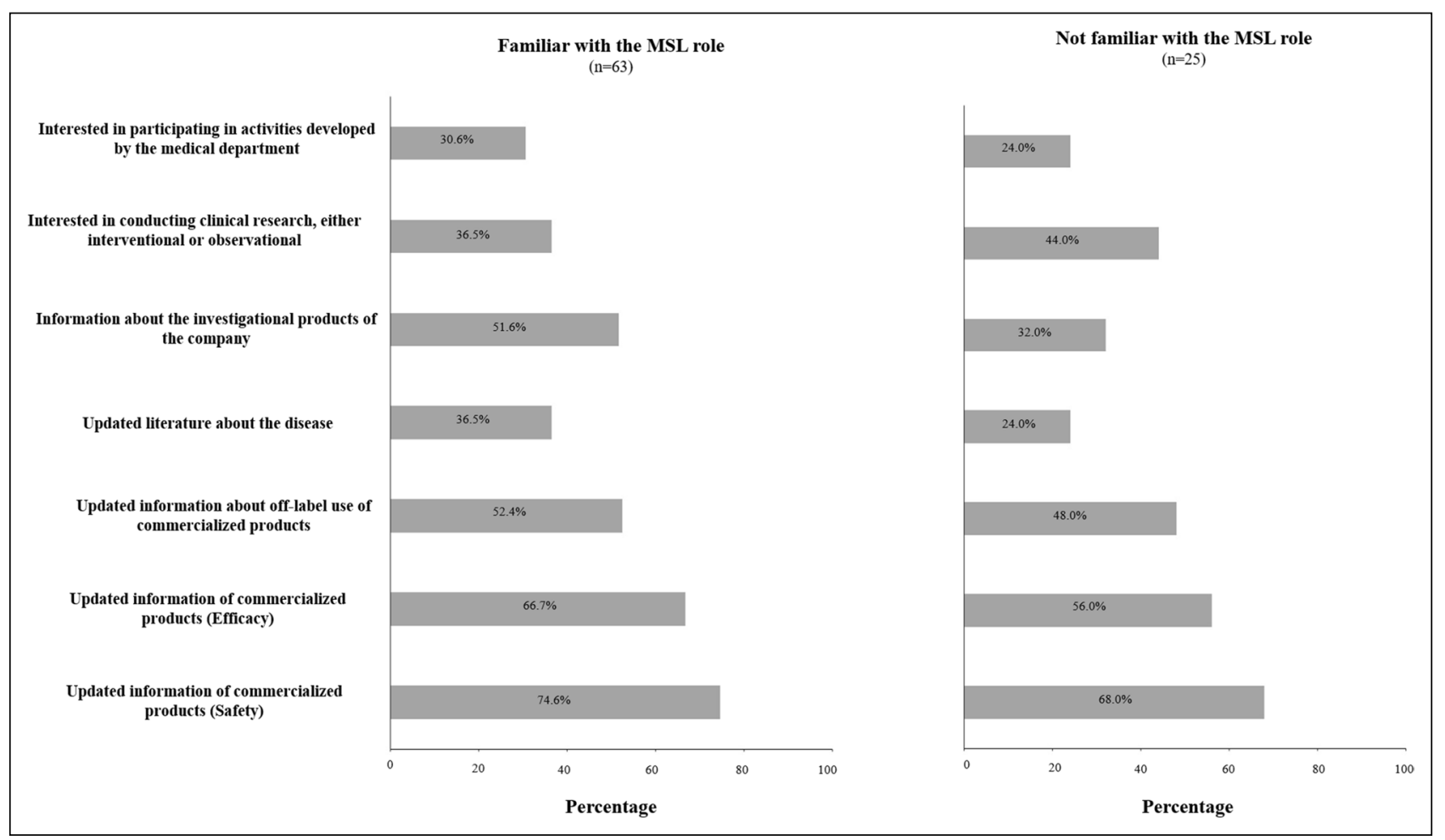

Fig. 3 Information exchanged during the HCP's meetings with the MSL

KOLs and HCPs have turned more virtual [19]. Participants in the 2020 online survey participated in one visit (31\%), two visits (12\%), or three visits (7\%).

It is necessary to remark the very positive opinion that HCPs/KOL have on the MSL, in terms of credibility and added value. However, there is room for improving this opinion and it could be related with the discussions about how the MSLs should evolve in the future, and what new responsibilities should be assumed. In our previous survey conducted among the MSLs in Spain, it was noted that MSLs should enhance some responsibilities, such as management of clinical trials and IIT's (68\%), and assume new ones such as involvement in the elaboration for National strategy (67\%), pharmacoeconomic knowledge (54\%), or managing budget (43\%) [18]. The findings by Chicharro et al. also support these results [15]. The authors stated that the complexity of the role was increasing, with a wide range of responsibilities apart from KOL's engagement and scientific exchange (i.e., company trial support, investigatorinitiate trial research). Moreover, since the introduction of new drugs relies on value-based pricing, MSLs can play a crucial role in market access, sharing not only clinical data but also patient outcome data and health economics outcomes research [20].

Finally, we found that more than a quarter of the respondents still do not know of the MSL role. Among them, 50\% could meet the KOL definition, which leads us to ask ourselves why these HCPs/KOLs were not aware about the role. One hypothesis could be that they are HCPs who are working in a local environment where the MSL are not able to reach due to the size of the teams. In addition, we have found a good opportunity for MSLs since $60 \%$ of HCPs previously unaware of MSLs are interested in meeting with the MSL, so this issue should be addressed. A solution to this could be a virtual meeting, which have been enhanced during the pandemic and are now well established as part of the MSL role.

The main limitation of our study derives from the methodology, especially from the subjective nature of surveys, providing only the opinion of a sample of subjects. Moreover, despite being one of the largest cohorts in this sort of studies, a higher number of participants could have facilitated the statistical analysis (comparisons between groups) and strengthen the conclusions. Available medical specialties were also limited by the participating scientific societies. Therefore, the methodological design of the study could have limited the sample size. Besides this, reported opinions were in line with those found in similar surveys [13, 15]. One of the reasons for the low participation among SEOM members was possibly due to the use of a Newsletter for informing their members about the availability of the survey. By contrast, one of the strengths of the study was the participation of the Spanish scientific societies. It avoided potential biases related with the recruitment of participants because 
no pharmaceutical company was involved in such a process. This fact was in contrast with other studies [13], in which companies were responsible for recruiting the participants. Furthermore, the fact that boards of directors of the scientific societies evaluated the survey and approved the publication for their members improved the quality and objectivity of our data. Another difference with previous literature is that we have not just described the profile of the MSL, but we have tried to identify characteristics in HCPs associated with the knowledge and value of the MSL role.

\section{Conclusion}

Our study demonstrates that the MSL role is well-known by the HCPs, mostly KOLs, and they agree on the importance of working together in scientific projects such as disease awareness, medical training, or clinical trials. Given the advance of targeted therapies and the move toward a personalized medicine, the MSL role will become more in demand and beneficial for HCPs in the years to come.

\section{Author contributions}

All authors contributed to the design of the work, the data acquisition, its interpretation, manuscript revision, and they have read and approved the submitted version.

\section{Funding}

No special funding was received for this article. Authors would like to thank to Meisys (pharmaceutical consultancy, Madrid, Spain) for the support in writing the article.

\section{Declarations}

\section{Conflict of interest}

The authors of this publication have no conflicts of interest to disclose. They are not speaking on behalf their actual companies or any previous companies.

\section{References}

1. Medical Science Liaison Society. What is a Medical Science Liaison? https://www.themsls.org/what-is-an-msl. Accessed 13 Oct 2020.

2. Medical Science Liaison Society. History and purpose of MSL guidelines. https://www.themsls.org/msl-guidelines/. Accessed 13 Oct 2020.
3. Moss RJ, Smith EB, Anderson G, et al. A survey of key opinion leaders to support curriculum development in advanced medical science liaison training. Ther Innov Regul Sci. 2015;49(1):45-9.

4. Morgan DK, Domann DE, Collins GE, et al. History and evolution of field-based medical programs. Drug Inf J. 2000;34(4):1049-52.

5. Baker DL. The role of the medical science liaison in industry. AORN J. 2010;91(3):394-8.

6. Koot D, McMaster A, Nel M, et al. Medical science liaisons (MSL) in Africa: a perspective. Pan Afr Med J. 2019;33:313.

7. Lawrence KR. Journey to the pharmaceutical industry and back: my experience as a medical science liaison. Am J Health Syst Pharm. 2002;59(21):2098-9.

8. Marrone CM, Bass JL, Klinger CK. Survey of medical liaison practices across the pharmaceutical industry. Drug Inf J. 2007:41:457-70.

9. Marrone CM, Bass JL, Klinger C. Survey of medical liaison practices No. 2: assessing training techniques and demonstrating value of medical Liaisons. Drug Inf J. 2008;42(1):67-80.

10. Bass JL, Marrone CM, Klinger CK. Surveys of medical liaison practices across the pharmaceutical industry: a review. Drug Inf J. 2009;43:685-95.

11. Bass JL, Marrone CM, Klinger CK. Survey of medical liaison practices 3: assessing practice trends across the pharmaceutical industry. Drug Inf J. 2010;44(5):535-49.

12. Klinger CJ, Marrone CM, Bass JL. Medical liaison survey 4 assessing tools used by medical liaisons, clinical trial involvement, and career strategies. Ther Innov Regul Sci. 2010;44:551-67.

13. Moss RJ, Black J. Health care professionals' expectations of the medical science liaison: a blinded survey. Ther Innov Regul Sci. 2013;47(2):203-8.

14. Jandhyala R. Personal selling and its effectiveness in generating sales: an assessment of: 'promotional', 'non-promotional' personal selling and 'non-personal' selling as part of the promotional mix in the pharmaceutical industry. ReseachGate; 2015. https:// ww.researchgate.net/publication/282876359

15. Chicharro A, Losada E, Marin H, et al. A survey of medical scientific liaisons in the pharmaceutical industry in Spain. J Med Market. 2017;16(1):4-9.

16. Suvorov N, Karaseva V, Stukalina E, et al. Medical science liaisons in real-world evidence studies: experience of AstraZeneca Russia. Ther Innov Regul Sci. 2018;52(1):57-61.

17. Tomiyasu M, Hayashi K, Moritsugu Y, et al. Proposal of standard for medical science liaison (MSL) profession in Japan: a viewpoint from the Japanese Association of Pharmaceutical Medicine (JAPhMed). Pharmaceut Med. 2020. https://doi.org/10.1007/ s40290-020-00355-8

18. Sastre V, Matesanz-Marín A, García C, et al. The medical science liaison role in Spain: a nationwide survey. Perspect Clin Res. 2020 (Accepted for publication).

19. The Medical Science Liaison Society. How KOLs prefer to engage with MSLs during the COVID-19 pandemic. https://www.thems ls.org/covid-19-kol-engagement-survey/

20. Mike Menta. Medical Affairs' changing role in market access and reimbursement. https://www.pharmaceuticalcommerce.com/opini on/medical-affairs-changing-role-in-market-access-and-reimb ursement/ 